\title{
Proteogenomics reveals molecular subtypes
}

$\begin{array}{ll}\text { These studies } & \begin{array}{l}\text { Despite extensive characterization of } \\ \text { the molecular features of non-small- } \\ \text { cell lung adenocarcinoma (LUAD), } \\ \text { the molecular characteristics } \\ \text { provide an } \\ \text { in-depth }\end{array} \\ \text { of LUAD specifically in patients of } \\ \text { East Asian ethnicity remain largely } \\ \text { the molecular } \\ \text { characteristics } \\ \text { of LUAD.... }\end{array} \quad \begin{aligned} & \text { integrative proteomic studies provide } \\ & \text { a detailed characterization of LUAD } \\ & \text { in this population, including in } \\ & \text { never-smokers with early stage disease. } \\ & \text { Researchers conducted indepen- } \\ & \text { dent integrated 'omics' analyses } \\ & \text { (including genomics, proteomics and } \\ & \text { phosphoproteomics information) } \\ & \text { of samples from }>100 \text { Chinese or } \\ & \text { Taiwanese patients. } \\ & \text { Minjia Tan, a senior author on the } \\ & \text { Chinese study explains the rationale } \\ & \text { for this approach: "Proteins are the } \\ & \text { functional executors of the cell, } \\ & \text { therefore, we conducted an in-depth } \\ & \text { characterization of the proteome } \\ & \text { and phosphoproteome of LUAD } \\ & \text { that will lay a foundation for a more } \\ & \text { comprehensive understanding of the } \\ & \text { molecular mechanisms of the disease." } \\ & \text { These investigators initially } \\ & \text { observed more EGFR alterations } \\ & \text { (in } 50 \% \text { of samples) and fewer } \\ & \text { KRAS alterations (6\%) compared } \\ & \text { with the TCGA cohort (14\% and } \\ & \text { 33\%, respectively). These findings } \\ & \text { confirm known distinctions in } \\ & \text { LUAD genotypes among patients of } \\ & \text { East Asian ethnicity relative to their } \\ & \text { western counterparts. } \\ & \text { Clustering analysis of the } \\ & \text { 25\% most variably expressed }\end{aligned}$

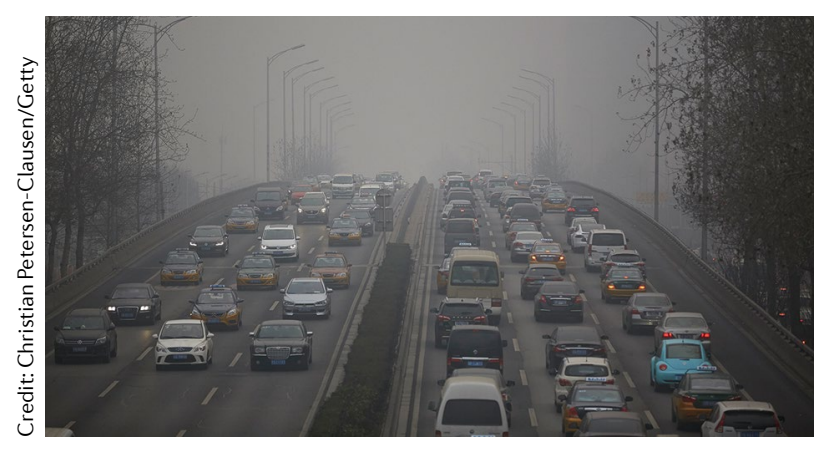

proteins revealed three distinct LUAD subtypes, with different prognostic implications. Subtype I, with overexpression of proteins involved in the regulation of tumour microenvironment and metabolism, was associated with the best prognosis, whereas subtype III, featuring overexpression of genes associated with proliferation and the proteasome, conferred the worse prognosis. Application of clustering analysis to the phosphoproteome also revealed three distinct subtypes: the overall level of concordance between phosphoproteomic and proteomic subtypes was limited (38\%), although proteomic subtype III had a good level of agreement with the equivalent phosphoproteomic subtype. Further investigations revealed robust negative associations between prognosis and circulating levels of HSP90 $\beta$, which were subsequently confirmed in a test cohort of 499 patients with LUAD.

Tan summarizes "Our proteomebased stratification revealed three subtypes related to different clinical and molecular features. Based on proteomics data and clinical information, we further nominated potential drug targets and validated the plasma protein level of HSP90 $\beta$ as a potential prognostic biomarker".

In a second integrated omics analysis, researchers from Taiwan investigated samples from 103 patients. This cohort comprised predominantly never-smokers (85 patients) with early stage non-small-cell lung cancer (NSCLC) (including 89 stage I-II NSCLCs, of which 80 were LUADs). Senior author Yu-Ju Chen highlights "LUAD in East Asia is considered a distinct disease characterized by a high incidence in never-smokers (>90\% of female patients in Taiwan), an earlier onset (5-10 years earlier than in other populations) and a high frequency of EGFR driver alterations (60\%). However, the genetic background, aetiology and risk factors for never-smokers remain to be understood."

Genomics analyses confirmed a high frequency of EGFR alterations (85\%). Further interrogation of these data revealed the presence of an APOBEC-high mutagenesis signature in $44 \%$ of patients, which was associated with younger age (mean age 52.9 years versus 66.3 years in those lacking this signature; $P=0.038$ ) and female gender (present in $57 \%$ of women versus $25 \%$ of men; $P=0.0045)$. A subset of patients with APOBEC-high tumours who received combination immune-checkpoint inhibition had prolonged progressionfree survival, suggesting that APOBEC is a candidate biomarker of response to these agents.

Consensus proteomic clustering revealed five distinct subtypes: notably, some patients with early stage disease had a proteomic subtype suggestive of a later-stage tumour. These features might provide early biomarkers of high-risk disease.

Pan-Chyr Yang, the lead oncologist in this study, summarizes "Owing to the multi-layer proteogenomic information revealed here, we were able to identify demographically distinct signatures of oncogenesis and progression in never-smokers and provide new insights into the management of early stage LUAD in this distinct group of patients."

These studies collectively provide an in-depth analysis of the molecular characteristics of LUAD and demonstrate the existence of considerable heterogeneity, even among a single histological subtype.

Peter Sidaway

ORIGINAL ARTICLES Xu, J. Y. et al. Integrative proteomic characterization of human lung adenocarcinoma. Cell 182, 245-261 (2020) | Chen, Y. J. et al. Proteogenomics of non-smoking lung cancer in East Asia delineates molecular signatures of pathogenesis and progression. Cell $182,226-244$ (2020) 